\title{
Time of Flight Mass Spectroscopy of Recoiled Ions Studies of Gallium Nitride Thin Film Deposition by Various Molecular Beam Epitaxial Methods
}

\author{
E. Kim ${ }^{1}$, I. Berichev ${ }^{1}$, A. Bensaoula ${ }^{1}$, A. Schultz ${ }^{2}$, K. Waters ${ }^{2}$ and W. Zagozdzon-Wosik ${ }^{3}$ \\ ${ }^{1}$ The Space Vaccum Epitaxy Center, University of Houston, \\ ${ }^{2}$ Ionwerks, \\ ${ }^{3}$ Department of Electrical and Computer Engineering, University of Houston,
}

(Received Tuesday, June 16, 1998; accepted Tuesday, September 15, 1998)

\begin{abstract}
Gallium Nitride (GaN) thin films were successfully grown by electron cyclotron resonance molecular beam epitaxy (ECR-MBE), gas source MBE (GSMBE), and chemical beam epitaxy (CBE). Time of flight mass spectroscopy of recoiled ions (TOF-MSRI) and reflection high energy electron diffraction (RHEED) were used in-situ to determine the surface composition, crystalline structure, and growth mode of GaN thin films deposited by the three MBE methods. The substrate nitridation and the buffer layers were monitored and optimized by TOF-MSRI and RHEED. For GSMBE, the gallium to nitrogen ratio is found to correlate well with ex-situ optical properties. In the case of CBE, carbon incorporation determines the surface morphology, crystalline quality and optical activity of the epilayers.
\end{abstract}

\section{Introduction}

\subsection{Time of Flight Mass Spectroscopy of Recoiled Ions}

With many interesting and useful applications, GaN has become one of the most important optoelectronic materials. Among the GaN thin film growth methods, molecular beam epitaxy (MBE) growth has become a recognized technique for fabricating state-of-the-art short wavelength opto-electronic devices, although metal-organic chemical vapor deposition currently remains the technology of choice for producing the majority of GaN-based devices. [1], [2] Nevertheless, $\mathrm{MBE}$ is expected to be superior in terms of composition, thickness, doping, and interface control due to the beam nature of the source fluxes and the availability of various in-situ monitoring tools. In addition, the lower growth temperatures and the absence of residual impurities in the process should allow hetero-structures with controllable doping profiles, lower defect densities.

Although GaN LED and laser diodes have been developed recently, the precise growth mechanism and the role of substrate nitridation and buffer layers are not yet clearly understood. Both substrate nitridation and buffer layer growth dramatically improve the quality of the GaN layers on the highly mismatched sapphire sub- strate. [3], [4] It is generally accepted that the nitridation step provides a better template for GaN nucleation and the thin low temperature GaN or AIN buffer layers relax the interfacial stress.

Ion scattering spectroscopy (ISS) is a well-established probe based on ion-solid interactions. A variant, called low energy ion scattering (LEIS) by time-of-flight (TOF) detection, is a powerful technique for surface structural and elemental analysis. [5] This technique has been proven as a real time thin film growth monitor in background pressures up to $10^{-2}$ torr [6], [7] and, therefore, can be particularly useful for studies of gas source MBE growth of GaN. It does not induce any measurable damage in the thin films due to the extremely low ion beam current densities used (as low as $\sim 10^{10}$ primary ions $/ \mathrm{cm}^{2}$ ). [8] The technique is essentially nondestructive. Since TOF-LEIS spectra are nearly independent of the chemical environment, there is a minimum matrix effect in contrast to secondary ion mass spectroscopy (SIMS). A recently developed ISS technique, known as mass spectroscopy of recoiled ions (MSRI), has a better sensitivity $\left(10^{4}\right.$ times higher for nitrogen detection) to light elements than SIMS, and superior minimum detection levels of $\sim 10^{17}$ ions $/ \mathrm{cm}^{3}$. [9] In addition, the low incidence angle of TOF-MSRI 
makes it suitable for real time surface analysis in an MBE environment. As a surface probe, TOF-MSRI is an ideal in-situ complementary technique to RHEED.

GaN thin films were grown in our laboratory by different MBE techniques with various $\mathrm{Ga}$ and $\mathrm{N}$ sources. At first, ECR plasma assisted MBE technique was employed. In this method, activated nitrogen gas was supplied from an ECR plasma source. Elemental Ga was evaporated from a Knudsen cell. Next, gas source MBE GaN deposition was investigated using $\mathrm{NH}_{3}$ and $\mathrm{Ga}$. There is no need for excitation of the source molecule in this method. Ammonia gas is easily dissociated on the hot sample surface. Finally, chemical beam epitaxy (CBE) was utilized. In the CBE method, triethyl gallium (TEG) and ammonia were used as precursors.

\section{Experimental Procedure}

\subsection{Sample Preparation}

GaN thin films are grown on 0.01-0.02 inch thick, 1inch diameter basal plane sapphire single crystal wafers. Before introduction into the load lock chamber, the samples are prepared in the following fashion. They are degreased with tri-chloroethylene, acetone and methanol, and rinsed with doubly de-ionized water. The substrates are then chemically polished in hot $3 \mathrm{H}_{2} \mathrm{SO}_{4}: 1$ $\mathrm{H}_{3} \mathrm{PO}_{4}$ by volume ratio in an acid mixture, rinsed in deionized water and nitrogen blow-dried.

\subsection{Thin Film Growth and In-Situ Analysis}

After this wet cleaning, the samples are mounted on an indium free 1-inch diameter sample holder. The samples are inserted into the introduction chamber where they are left until the pressure drops to the $10^{-7}$ torr range. The sample is then transferred to the manipulator in the main chamber where it is annealed in vacuum to $850{ }^{\circ} \mathrm{C}$ for 30 minutes. This process allows removal of residual hydrocarbons, oxides, and most other contaminants from the sample surface. This thermal cleaning is monitored by TOF-MSRI and RHEED. In this manner, the crystal structure and composition are known before each growth. In particular, the $\mathrm{H}, \mathrm{Al}, \mathrm{O}, \mathrm{C}$ and any other impurities are monitored by TOF-MSRI. At this point, the sample's temperature is lowered to that appropriate to nitridation $\left(600-750{ }^{\circ} \mathrm{C}\right.$ ), and either ammonia or ECR- $\mathrm{N}_{2}$ is let into the chamber. Following nitridation, a low temperature buffer layer is deposited at $450-550{ }^{\circ} \mathrm{C}$ with either ECR-MBE (ECR-N 2 and Ga), GSMBE $\left(\mathrm{NH}_{3}\right.$ and $\mathrm{Ga}$ ), MOMBE (ECR-N ${ }_{2}$ and TEG), or CBE $\left(\mathrm{NH}_{3}\right.$ and TEG). Finally the sample is heated to the appropriate final epilayer growth temperature (750-850 ${ }^{\circ} \mathrm{C}$ ) and the epilayer deposition is initiated. At the end of the growth, the sample's temperature is lowered gradually under a group $\mathrm{V}$ over-pressure. If no post growth in-situ characterization experiments are planned, the sample is removed from the reactor.

\section{Results and Discussions}

\subsection{ECR MBE}

The ASTeX ${ }^{\circledR}$ compact ECR source was developed specifically for MBE applications. The ECR can be operated in a vacuum environment in the $10^{-5}-10^{-4}$ torr range. In this range, the mean free path is about 1 meter, which is much longer than the source to substrate distance thus molecular flow regime is maintained. With ECR nitrogen source, degradation of the hot effusion cells and PBN sample heater is significantly lower than in case of ammonia.

Various energetic nitrogen species $\left(\mathrm{N}_{2}{ }^{*}, \mathrm{~N}_{2}{ }^{+}, \mathrm{N}\right.$ and $\mathrm{N}^{+}$) are generated in an ECR source. The effect of ion damage during $\mathrm{MBE}$ growth of $\mathrm{GaN}$ using plasma sources of active nitrogen is well documented. Several methods have been attempted to reduce or even eliminate this effect. For plasma sources there are: positive bias of substrate or grid, external transverse magnetic field, and modified ECR plasma exit aperture. [10], [11] Using this method, GaN films at growth rate as high as $1 \mu \mathrm{m} / \mathrm{hr}$ were obtained. [12]

\subsubsection{Substrate Nitridation}

Sapphire $\left(\mathrm{Al}_{2} \mathrm{O}_{3}\right)$ substrates were nitridated under ECR nitrogen plasma for 20-30 minutes to create an AlN template for subsequent $\mathrm{GaN}$ nucleation. The oxygen atoms in the topmost layers can be substituted by the nitrogen atoms supplied by the nitrogen ECR plasma source. For these experiments, the applied nitrogen gas flow was $3 \mathrm{sccm}$ and the corresponding chamber pressure was $1.7 \times 10^{-4}$ torr. The microwave power was 200-250 W and the current setting on the electromagnet power supply was 17-18 A. In a standard configuration, the ECR was fitted with a 3/8-inch diameter boron nitride (BN) aperture placed at the source exit. Such an aperture increases the pressure in the plasma region and allows easy ignition and stable plasma condition.

First, the nitridation efficiency was investigated as a function of sample temperature. A thermally cleaned sapphire substrate was nitridated for 10 minutes at each temperature. The temperature was varied between 550$850{ }^{\circ} \mathrm{C}$. A TOF-MSRI spectrum was taken after each exposure. The TOF-MSRI results, the relative peak intensity (surface coverage) of the surface atoms, are presented in figure 1. A weak nitrogen peak was detected above $650{ }^{\circ} \mathrm{C}$ and increased when the sample was further nitridated at $700{ }^{\circ} \mathrm{C}$. The onset of nitridation of sapphire was only identified by RHEED for a sample temperature above $700{ }^{\circ} \mathrm{C}$. TOF-MSRI is thus a more 
sensitive probe to optimize the substrate nitridation process.

While the previous experiment allowed determination of the optimum nitridation temperature, in the following we investigated the effect of exposure time at a constant ECR power of $200 \mathrm{~W}$ and a substrate temperature of $700{ }^{\circ} \mathrm{C}$. We did not find degradation of nitridated surface - partially covered $\mathrm{Al}_{2} \mathrm{O}_{3}$ by crystalline AlN when substrate surface was nitridated at higher temperature determined by in-situ RHEED and TOF-MSRI. However, usually we perform nitridation at the minimum required temperature to avoid unnecessary heat supply that may reduce sample heater's lifetime. Previous reports suggest that too short or too long a nitridation time resulted in the deterioration of the $\mathrm{GaN}$ epilayer quality. [3] To that end, an annealed sapphire surface was exposed to the ECR source, and the TOFMSRI spectra were taken at 10-min intervals. In figure 2, the TOF-MSRI spectra at each interval are plotted. After a 20-min exposure time, the N signal is saturated. The carbon signal, however, continues to decrease with exposure, which implies ECR plasma is effective not only for the nitridation but also for cleaning a substrate. RHEED showed that an overlapped diffraction pattern of $\mathrm{AlN}$ and $\mathrm{Al}_{2} \mathrm{O}_{3}$ due to a thin $\mathrm{AlN}$ over layer on the sapphire substrate. A nitridated AIN layer remained as a single crystal up to 30 -min exposure. No significant change is seen after a 30-min ECR plasma exposure by in-situ measurements.

\subsubsection{Buffer Layer}

Low temperature GaN buffer layers were grown by ECR-MBE at various growth temperatures. Typical deposition time for these buffer layers was 30 minutes, which resulted in GaN layer thickness in the range of 300-500 A. RHEED from these layers show single crystalline surfaces for a growth temperature of $500{ }^{\circ} \mathrm{C}$.

The streaky RHEED patterns were obtained, and they were remained unchanged when these layers are annealed to $750{ }^{\circ} \mathrm{C}$ under an ECR-N 2 beam. Also the buffer layers' crystallinity seem to be insensitive to the substrate nitridation time for $30 \mathrm{~min}$. TOF-MSRI was utilized to survey the surface composition of the sapphire substrate after nitridation and during buffer layer growth. Figure 3 shows the elemental composition at four different times. The nitridated surface shows only $\mathrm{N}, \mathrm{O}$, and $\mathrm{Al}$ and no other contaminants. Immediately following the start of the buffer layer growth, the Al signal drops significantly. The buffer layer seems to proceed in a two dimensional growth fashion. Within five minutes, the $\mathrm{Al}$ and $\mathrm{O}$ signals completely disappear. The buffer layer has covered the entire substrate, and its thickness is greater than a few monolayer (the ion probe depth range). The TOF-MSRI spectrum taken after 30 min does not show a significant difference. It remains also unchanged after the annealing at $750{ }^{\circ} \mathrm{C}$.

\subsection{Gas Source MBE}

In this technique, we used ammonia gas as the group $\mathrm{V}$ nitrogen source and elemental $\mathrm{Ga}$, from a heated effusion cell, for the group III material. Using this method, single crystal GaN thin films with growth rates as high as $7500 \AA / \mathrm{hr}$ were obtained. The growth rate and the crystalline quality, as well as the optical and electrical properties of these thin films, were extremely dependent on the $\mathrm{Ga} / \mathrm{NH}_{3}$ flux ratios, the substrate temperature, and the pre-deposition treatment. Below, we will summarize the results in optimizing the above parameters using TOF-MSRI and RHEED in-situ and PL ex-situ.

\subsubsection{Substrate Nitridation}

Following the sample's introduction into the growth chamber, the substrate surface was exposed to a nitridation step. In GSMBE, the nitridation is performed by exposing the clean sapphire substrate to an ammonia beam. The nitridation process depends on the substrate temperature and the exposure time. Similarly to ECRMBE growth, TOF low energy ion scattering MSRI and RHEED confirmed the surface nitridation. Using ammonia, the onset of nitridation occurs at a surface temperature of $780{ }^{\circ} \mathrm{C}$. At this temperature, a small nitrogen peak is detected by TOF-MSRI. The nitrogen signal is quite small, when compared to the case of ECR-MBE nitridation. The ammonia nitridation is clearly less effective than energetic $\mathrm{N}$ species. The need for thermal dissociation of ammonia requires nitridation at elevated temperatures $\left(>780^{\circ} \mathrm{C}\right)$. Such a high temperature limits the residence times of the reactants, and results in desorption of the $\mathrm{N}$ species before the nitridation reaction can occur. Such a reaction also requires an $\mathrm{O}$ to $\mathrm{N}$ exchange step, which further reduces the nitridation reaction probability when using ammonia. The oxygen peak, as measured by TOF-MSRI, is not significantly changed with nitridation time. This supports the argument that very little $\mathrm{O}$ to $\mathrm{N}$ exchange has occurred. Furthermore, RHEED images acquired during nitridation show a transition from a 2D-diffraction pattern (from an ordered sapphire surface) to a diffused image-no clear spacing related to an AlN over-layer was observed. These results agree with a previous report claiming that an amorphous phase aluminum oxy-nitride (AlON) layer was observed after ammonia nitridation of sapphire during MOCVD growth. [13]

\subsubsection{Buffer Layer}

In our experiments, the buffer layers were typically grown by GSMBE at a substrate temperature of $550{ }^{\circ} \mathrm{C}$. An immediate observation was that $\mathrm{GaN}$ film deposition occurred below the previously measured ammonia decomposition temperature $\left(780^{\circ} \mathrm{C}\right)$. The availability 
of Ga or a GaN surface seems to enhance the reactivity of the ammonia precursor. [14] It is apparent that this $\mathrm{GaN}$ layer does not fully cover the substrate since both the $\mathrm{Al}$ and $\mathrm{O}$ peaks still appear on the spectrum.

RHEED analysis of these GaN buffer layers shows diffuse and very dim patterns such as those from near amorphous and rough surfaces. The buffer layer's crystal ordering and surface morphology improve with annealing temperature. TOF-MSRI data taken during this process show a significant change in the $\mathrm{Ga} / \mathrm{N}$ ratio (from 5.4/1 to less than 2.5/1). The change in the $\mathrm{Ga} / \mathrm{N}$ ratio is exclusively due to a decrease in the Ga signal. This is interpreted as desorption of the excess elemental $\mathrm{Ga}$ from the low temperature GaN layers. The excess $\mathrm{Ga}$ is expected to reduce the crystallinity of the material and, through formation of gallium droplets, significantly increases the surface roughness. The annealing results in a crystalline surface with superior morphology. Low energy electron diffraction (LEED) studies, on amorphous GaN buffer layers grown by MOCVD, have reported that post-growth re-crystallization occurs during annealing. [15]

\subsubsection{In-Situ Growth Optimization}

Prior to optimizing the growth of thick GaN layers, we first undertook a series of experiments investigating the relationship between the surface stoichiometry and the ammonia and gallium fluxes. Intuitively, one should expect that the more stoichiometric the surface, the better the epitaxy. Our main concern, however, was to determine the initial growth conditions which allow the best $\mathrm{Ga} / \mathrm{N}$ ratio while keeping the lowest possible flow of corrosive ammonia. TOF-MSRI was utilized to measure in-situ the $\mathrm{Ga} / \mathrm{N}$ ratio during $\mathrm{GaN}$ deposition.

The dotted curve in figure 4 presents data for a substrate temperature at $800^{\circ} \mathrm{C}$ and a Ga cell temperature at $950{ }^{\circ} \mathrm{C}$ (beam equivalent pressure (BEP) of $2.8 \times 10^{-6}$ torr). The ammonia pressure is decreased from above $1.0 \times 10^{-4}$ torr to less than $5.0 \times 10^{-6}$ torr as measured by a flux gauge in the growth chamber. Under these conditions, we find that the $\mathrm{Ga} / \mathrm{N}$ ratio increases rapidly for either too high or too low flows. There is, however, a large window where a near unity $\mathrm{Ga} / \mathrm{N}$ peak intensity ratio can be easily maintained. The increase for low ammonia flows can be easily understood through a simple surface depletion argument. That at high flows is more difficult to interpret. RHEED patterns from these surfaces do not show any obvious transition of surface structure. We hypothesize that the reaction by-product $\left(\mathrm{H}_{2}\right)$ should be effectively formed on the surface, and liberated for the completion of $\mathrm{GaN}$ synthesis. If the ammonia arrival flux is high, most surface area is occupied by source molecules. Above a certain threshold the number of available $\mathrm{H}$ atom adsorption sites becomes limited, and the $\mathrm{NH}_{3}$ decomposition rate is reduced. Consequently, the $\mathrm{Ga} / \mathrm{N}$ ratio increases for very high ammonia pressures

The solid curve in figure 4 also shows results from a similar experiment with the exception that the Ga cell temperature is increased by $50{ }^{\circ} \mathrm{C}$ to $1000{ }^{\circ} \mathrm{C}$. Two observations can be made: (i) the low $\mathrm{Ga} / \mathrm{N}$ window becomes extremely narrow with a minimum $\mathrm{Ga} / \mathrm{N}$ ratio of 2.5 (at $8.5 \times 10^{-6}$ torr) and (ii) the $\mathrm{Ga} / \mathrm{N}$ ratio for most of the pressure range is $\sim 5$. The low-pressure side explanation is similar to the previous case. The overall increase in the $\mathrm{Ga} / \mathrm{N}$ ratio can be explained simply by a larger equilibrium $\mathrm{Ga}$ surface population. There is, however, a narrow window where the $\mathrm{Ga} / \mathrm{N}$ is at a minimum. The ammonia surface dissociation and reaction must be very sensitive to the total surface coverage of source molecules. Too little surface Ga lowers reactivity due to the lack of enough number of $\mathrm{Ga}$ atoms for the reaction with $\mathrm{NH}_{3}$; too high $\mathrm{Ga}$ fluxes blocks intermediary reaction paths and lowers the incorporation rates.

\subsubsection{Correlation between TOF-MSRI and}

Photoluminescence

To further investigate the extent to which the GaN epilayer's properties depend on the low temperature buffer layer, we designed the following experiment. GaN thin films were deposited on sapphire with GSMBE at fixed growth conditions with the exception that the nitridation and buffer layer steps were different in each case. The final layers were all grown by GSMBE. At the end of each growth, TOF-MSRI was utilized to measure the surface stoichiometry. Figure 5 clearly displays a relationship between the $\mathrm{Ga} / \mathrm{N}$ ratio, the oxygen impurity level, and the photoluminescence (PL) full width half maximum (FWHM) and intensity.

The closer to stoichiometry the surface, the better the PL result. While at first, a sample $(\mathrm{Ga} / \mathrm{N}=3.0$ but FWHM $=158 \mathrm{meV}$ ) may seem to be an exception, a closer analysis of its MSRI spectrum shows a surface oxygen shoulder from either ammonia contamination or improper initial growth conditions. These results are significant for use of TOF-MSRI as a real time optimization tool for $\mathrm{GaN}$ materials development and control of optoelectronic device fabrication.

\subsection{Chemical Beam Epitaxy}

As in $\mathrm{GaN}$ metal organic chemical vapor deposition (MOCVD), high vapor pressure organo metallic precursors containing group III metals and hydride group V compounds are normally used for source materials in CBE thin film growth. The group III precursor gas lines were maintained at $35^{\circ} \mathrm{C}$.

\subsubsection{Nucleation}

Prior to thin film deposition, the substrate was nitridated using the ECR source. To that end, the sapphire sub- 
strate, at a temperature of $750{ }^{\circ} \mathrm{C}$, was exposed to the $\mathrm{N}_{2}$-ECR source for 10-15 minutes.

Initially CBE buffer layer growth was attempted using standard procedures with TEG and $\mathrm{NH}_{3}$ precursors. No clear GaN nucleation was observed on the surface at any growth temperature. The TOF-MSRI results from these initial studies are described in figure 6 . At temperatures below $550{ }^{\circ} \mathrm{C}$, only a weak MSRI Ga peak is observed. In the absence of a nitrogen signal, this indicates that deposition of Ga or coordination of TEG on the surface occurred. When the growth temperature is increased to $750{ }^{\circ} \mathrm{C}$ in order to enhance the ammonia surface dissociation, TOF-MSRI measurements again still revealed no GaN deposition. Only a very little nitrogen peak is found with absence of Ga signal. The previously deposited $\mathrm{Ga}$ was evaporated and an AlN overlayer was formed during this process. The presence of TOF-MSRI signals from the sapphire substrate (aluminum and oxygen) further indicates that coverage is incomplete under both conditions. The lack of $\mathrm{GaN}$ nucleation directly on the nitridated sapphire substrate most likely results due to the following reasons: (i) At these low temperatures the ammonia dissociation is minimal but, as shown in GSMBE, is enhanced by a Ga rich surface. In the case of a surface TEG or the intermediate diethyl gallium (DEG) molecule, such an enhancement might not occur due to a less favorable bonding configuration. (ii) The TEG surface binding energy, being much weaker than $\mathrm{Ga}$, can result in a low overall residence time of the TEG-NH 3 complex. Consequently the TEG- $\mathrm{NH}_{3}$ complex is less bound to the surface and desorbs. The surface residence time can be made lower if the TEG surface bond gets weaker upon reaction between TEG and $\mathrm{NH}_{3}$. (iii) Our experimental results show a very high oxygen content in these low temperature buffer layers. Considering that oxygen gets incorporated into the layers from an oxidized Ga precursor (for example, $\mathrm{GaR}_{3-\mathrm{X}}(\mathrm{OR})_{\mathrm{X}}$ with $\mathrm{X}=1,2,3$ ), where the gallium is less electron deficient than in TEG since the oxygen from the $\mathrm{Ga}-\mathrm{O}$ bond provides it with an electron. The oxidized Ga compounds then becomes less bound to the surface and desorbs more readily. In addition, the reaction intermediate, $\mathrm{NH}_{3}-\mathrm{GaR}_{3-\mathrm{X}}(\mathrm{OR})_{\mathrm{X}}$, is more difficult to form (higher activation energy is required) than TEG-NH${ }_{3}$.

Due to the difficulty of nucleating a GaN film on sapphire with CBE, we switched the group $\mathrm{V}$ nitrogen source from ammonia to ECR plasma activated nitrogen and deposited a low temperature buffer layer with $\mathrm{N}_{2^{-}}$ ECR using TEG as the group III precursor. This method is analogous to metal organic MBE (MOMBE) epitaxial growth. The $\mathrm{N}_{2}$-ECR plus TEG growths resulted in
GaN films at substrate temperatures as low as $450{ }^{\circ} \mathrm{C}$. The TOF-MSRI spectrum from these thin layers contains $\mathrm{Ga}$ and $\mathrm{N}$ peaks without any Al signal. The buffer layer is thus continuous and covers the entire substrate. Carbon and oxygen signals are also visible on the same spectrum.

The carbon peak originates from the group III organometallic precursor. Its intensity significantly decreases after a high temperature annealing. The oxygen signal is stable and remains constant. The oxygen incorporation is either due to surface diffusion from bulk or an impurity present during the deposition process. TOF-MSRI from either ECR MBE $\left(\mathrm{Ga}+\mathrm{N}_{2}\right)$ or GSMBE $\left(\mathrm{Ga}+\mathrm{NH}_{3}\right) \mathrm{GaN}$ thin films grown in the same reactor do not show any residual surface oxygen. Based on this fact, the oxygen most probably originates from the reaction of $\mathrm{O}_{2}$ with the TEG precursor. The result is a very stable alkoxide intermediate.

The oxygen is thus very efficiently delivered to the growth surface as a C-O bond. The carbon subsequently desorbs upon annealing leaving the oxygen behind. This is consistent as well with the lower strength of the $\mathrm{Ga}-\mathrm{C}$ bond relative to that of either $\mathrm{Ga}-\mathrm{N}$ or Ga-O.

\subsubsection{The Influence of Carbon Incorporation on Film Quality}

Following the initial deposition of crystalline low temperature GaN buffer layers, GaN thin films were deposited with CBE at growth rates from 1000-4000 ̊̊hr. For CBE growth of the final layers, the substrate temperature was varied from $700-850{ }^{\circ} \mathrm{C}$, and different TEG to ammonia flow ratios were investigated. The substrate temperature and precursors flow rates were varied in an effort to produce crystalline high purity films.

The carbon surface composition was monitored insitu by TOF-MSRI. Our results show that the incorporation of carbon is both temperature and $\mathrm{TEG} / \mathrm{NH}_{3}$ ratio dependent, but clearly, the substrate temperature is the most critical factor controlling crystalline quality. A clear correlation also exists between surface carbon composition and GaN crystallinity. Growths performed at a constant substrate temperature of $850{ }^{\circ} \mathrm{C}$, but with increasing TEG to $\mathrm{NH}_{3}$ flow ratio, yield thin films with higher surface $\mathrm{C}$ content and deteriorating crystalline quality (as determined by RHEED). In this study, we carefully investigated the growth temperature range between $700{ }^{\circ} \mathrm{C}$ and $850{ }^{\circ} \mathrm{C}$. Only polycrystalline films were grown below $750{ }^{\circ} \mathrm{C}$, but single crystalline layers were achieved for higher temperatures. At $750{ }^{\circ} \mathrm{C}$, both textured polycrystal and 3D transmission single crystalline RHEED diffraction features were observed. It is only in a narrow temperature window $\left(800-825^{\circ} \mathrm{C}\right)$ that 
two-dimensionally (2D) smooth single crystal layers were obtained.

A correlation between surface carbon composition and $\mathrm{GaN}$ crystallinity is further supported when growths at a constant substrate temperature of $\sim 850^{\circ} \mathrm{C}$, but using various TEG to $\mathrm{NH}_{3}$ flow ratios, yield the same results: as TEG flow (surface carbon) increases, crystallinity deteriorates. RHEED patterns from CBE GaN layers grown at different TEG flow rates showed deterioration of film crystallinity while TEG/NH $\mathrm{NH}_{3}$ flow ratio was increased. Both the surface carbon and hydrogen signal intensities increase with TEG flow.

\section{Conclusion}

The sapphire substrate nitridation process is clearly determined by TOF-MSRI, which is shown to be more sensitive to onset of nitridation than RHEED. In the case of ECR- $\mathrm{N}_{2}$ plasma nitridation, the optimum temperature and saturation of N/O peak intensity ratio was observed. ECR- $\mathrm{N}_{2}$ plasma nitridation is more effective for oxygen replacement than ammonia gas. ECR MBE resulted in continuous crystalline coverage buffer layer while noncrystalline discontinuous buffer layers were grown by GSMBE. In the case of CBE, low temperature nucleation was not observed, so that buffer layers were grown by MOMBE.

Gas source MBE, using ammonia and Ga, was utilized in GaN thin films growth. The chemical decomposition and incorporation of $\mathrm{NH}_{3}$ in the thin films were studied for different growth conditions. The nitridation of sapphire was demonstrated but was determined to be much more difficult to achieve than with an ECR plasma source. GaN epilayers grown with a low temperature buffer layer exhibited superior film quality. A direct correlation between in-situ TOF-MSRI and ex-situ PL results was observed. Samples with a low $\mathrm{Ga} / \mathrm{N}$ ratio in TOF-MSRI showed the best optical quality. The $\mathrm{NH}_{3}$ pressure during growth significantly influenced this $\mathrm{Ga} /$ $\mathrm{N}$ ratio.

TOF-MSRI identified that nucleation layers could not be deposited directly on the sapphire substrate using the CBE precursors. The use of TEG and ECR- $\mathrm{N}_{2}$ species allowed the growth of crystalline buffers. The growth temperature and $\mathrm{N}_{2}$ flow strongly affected the crystallinity of these buffer layers. Amorphous and polycrystalline buffer layers became island-like upon annealing to $900{ }^{\circ} \mathrm{C}$ in vacuum while the crystalline buffer layers remained unchanged. Similarly, the epilayers' crystal quality depended critically on the growth temperature and TEG flows. TOF-MSRI studies found that the residual carbon level is highly temperature dependent and influenced greatly the surface morphology and crystallinity (from RHEED). It is only within a narrow temperature window, $800-825^{\circ} \mathrm{C}$ that the carbon level is at a minimum. Within that range, $\mathrm{GaN}$ epilayers with strong band edge PL signals were obtained. The oxygen surface levels, as measured by TOF-MSRI, in all CBE GaN were high and did not decrease after annealing. It is postulated that an oxidized $\mathrm{Ga}$ compound in the TEG source is at the origin of such high levels.

\section{ACKNOWLEDGMENTS}

This work was supported by funds from a NASA cooperative agreement no. NCC8-127 to SVEC, a Texas Advanced Research Program Grant no. 1-1-27764, and a Texas Advanced Technology Program Grant no. 11-32061. Ionwerks would like to acknowledge SBIR contracts F33615-97-C-1035 monitored by J. Van Nostrand at Wright Patterson AFB and NASA contract NAS8-97011 monitored by J. Watkins at Marshall Space Flight Center.

\section{REFERENCES}

[1] S Nakamura, M Senoh, SI Nagahama, N Iwasa, T Yamada, T Matsushita, H Kiyoku, Y Sugimoto, T Kozaki, H Umemoto, M Sano, K Chocho, Appl. Phys. Lett. 72, 2014 (1998).

[2] S. Nakamura, M. Senoh, N. Iwasa, S. Nagahama, Jpn. J. Appl. Phys. 34, L797-L799 (1995).

[3] N Grandjean, J Massies, M Leroux, Appl. Phys. Lett. 69, 2071-2073 (1996).

[4] S. Nakamura, Jpn. J. Appl. Phys. 30, L1705-L1707 (1991).

[5] W. Eckstein, Nucl. Instrum. Methods B 27, 78 (1987).

[6] S. M. Gate, D. D. Koleske, Thin Sol. Films 225, 160 (1993).

[7] M. Aono, M. Katayama, E. Nomura, T. Chasse, D. Choi, M. Kato, Nucl. Instrum. Methods B 37, 264 (1989).

[8] T. M. Buck, "Methods of Surface Analysis", edited by A. W. Czanderna, Elsevier, Amsterdam, 1975

[9] M. S. Hammond, J. A. Schultz, A. R. Krauss, J. Vac. Sci. Technol. A 13, 136 (1995).

[10] R. Beresford, A. Ohtani, K. S. Stevens, M. Kinniburgh , J. Vac. Sci. Technol. B 13, $792-795$ (1995).

[11] R.J. Molnar, T.D. Moustakas, J. Appl. Phys. 76, 4587 (1994).

[12] I. Berishev, Esther Kim, A. Bensaoula, unpublished.

[13] K. Uchida, A. Watanabe, F. Yano, M. Kouguchi, T. Tanaka, S. Minagawa, J. Appl. Phys. 79, 3487-3491 (1996).

[14] S. S. Liu, D. A. Stevenson, J. Electrochem.Soc. 125 , 1161 (1978).

[15] J. N. Kuznia, M. Asif Khan, D. T. Olson, Ray Kaplan, Jamie Freitas , J. Appl. Phys. 73, 4700-4702 (1993). 


\section{FIGURES}

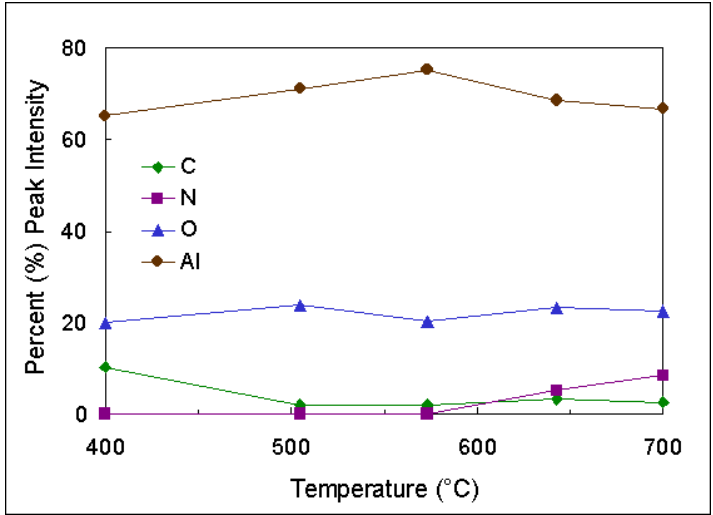

Figure 1. The change of surface elemental population of a sapphire substrate during ECR plasma nitridation. The relative peak intensities are obtained by TOF-MSRI at different surface temperature.

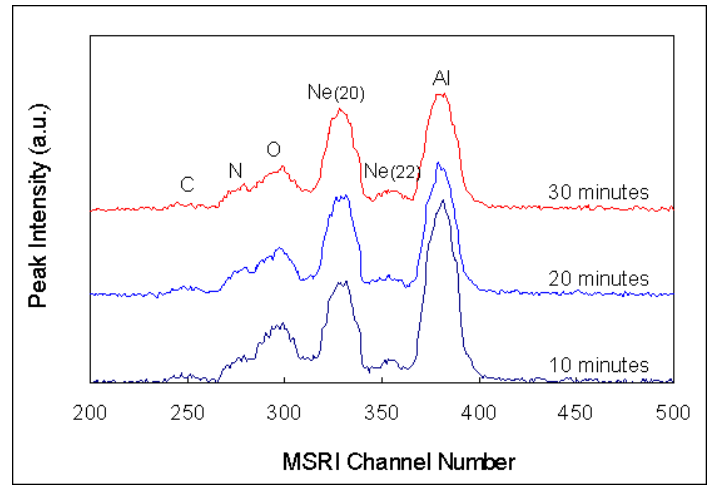

Figure 2. The TOF-MSRI spectra vs. ECR-nitridation exposure time from a sapphire surface. The ECR power is $200 \mathrm{~W}$ and the substrate temperature is $700{ }^{\circ} \mathrm{C}$.

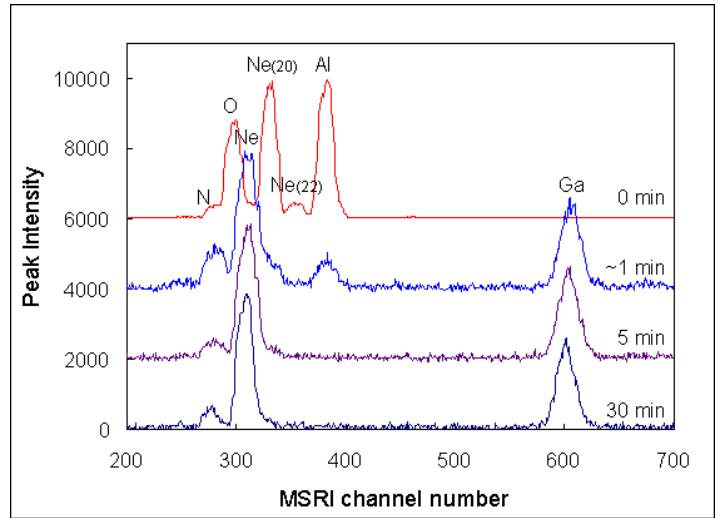

Figure 3. TOF-MSRI spectra during a GaN buffer layer deposition. The neon scatter peak position shifts to lower time of flight due to the ECR plasma operation during the second, third and fourth spectra acquisitions.

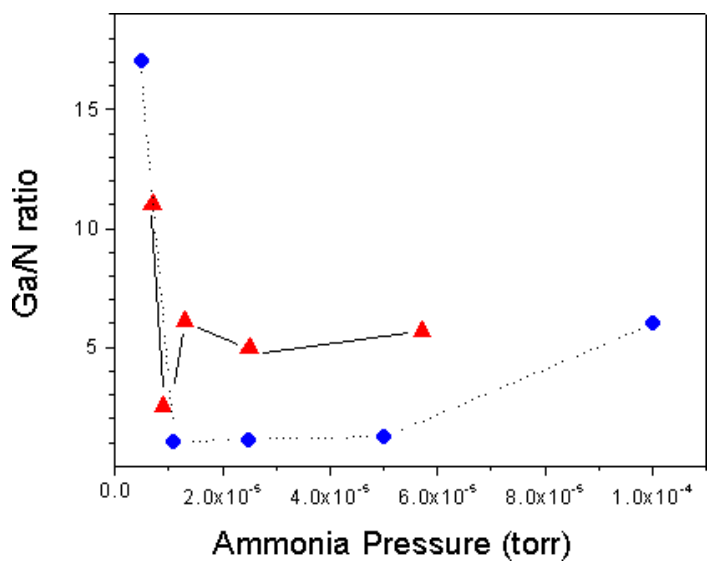

Figure 4. Ga to $\mathrm{N}$ peak intensities measured by TOF-MSRI during GSMBE growth of GaN under varying ammonia gas pressure. The dotted curve is obtained for a Ga cell of $950^{\circ} \mathrm{C}$. The solid curve is obtained for a Ga cell of $1000{ }^{\circ} \mathrm{C}$. In both cases, the substrate temperature is $800{ }^{\circ} \mathrm{C}$.

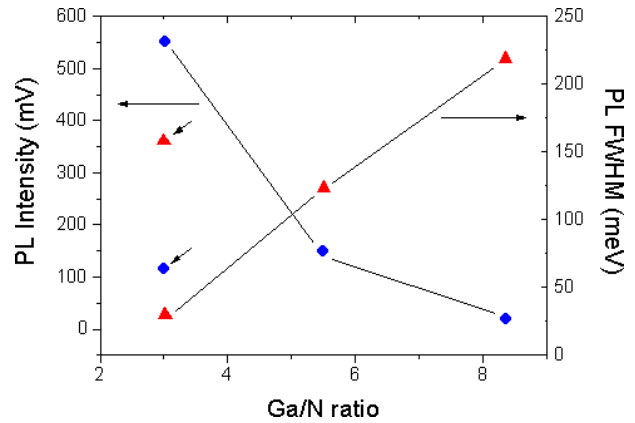

Figure 5. The correlation of optical properties of GSMBE GaN and $\mathrm{Ga} / \mathrm{N}$ peak intensity ratio as measured by TOF-MSRI. PL band edge peak intensity (red triangles) and FWHM of PL signal (blue circles) at $10 \mathrm{~K}$. A deviation is found for the sample containing oxygen as a contaminant by TOF-MSRI (marked by short arrows).

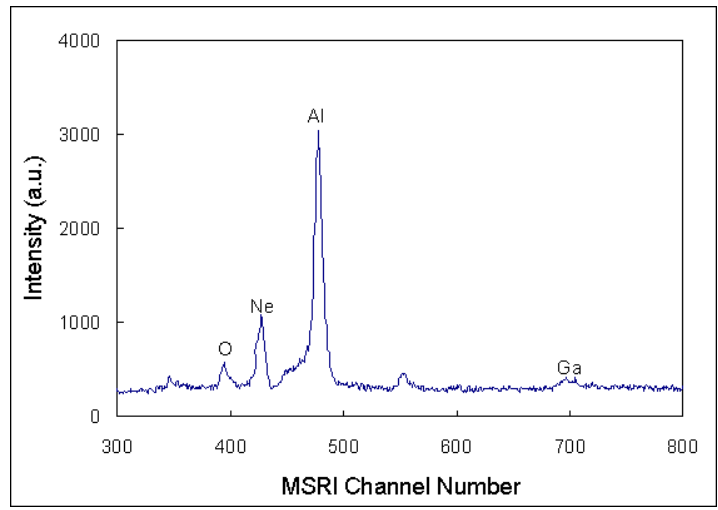

Figure 6. A TOF-MSRI spectrum from a sapphire substrate exposed to $\mathrm{NH}_{3}+\mathrm{TEG}$ at $550{ }^{\circ} \mathrm{C}$. Only a small Ga peak is seen in the absence of $\mathrm{N}$ peak. 